\title{
THE PHENOMENA OF THE TIME-VIOLATING PHOTON POLARIZATION PLANE AND NEUTRON SPIN ROTATION BY A DIFFRACTION GRATING. NEW METHODS OF MEASURING OF THE TIME-VIOLATING INTERACTIONS.
}

\author{
V.G.BARYSHEVSKY \\ Nuclear Problems Institute, Bobruiskaya Str 11, \\ Minsk 220080 Belarus. \\ Electronic address: bar@inp.belpak.minsk.by
}

\begin{abstract}
It is shown, that the new phenomena of the T-violating photon polarization plane and neutron spin rotation appear under diffraction in a noncentrosymmetrical diffraction grating. The equations describing the T-violating photon scattering by a diffraction grating and neutron interaction with noncentrosymmetrical crystal have been obtained. These phenomena may be used for time-violating interactions investigation.
\end{abstract}




\title{
THE PHENOMENA OF THE TIME-VIOLATING PHOTON POLARIZATION PLANE AND NEUTRON SPIN ROTATION BY A DIFFRACTION GRATING. NEW METHODS OF MEASURING OF THE TIME-VIOLATING INTERACTIONS.
}

\author{
V.G.BARYSHEVSKY \\ Nuclear Problems Institute, Bobruiskaya Str 11, \\ Minsk 220080 Belarus. \\ Electronic address: bar@inp.belpak.minsk.by
}

Since the discovery of the CP-violation in decay of K-mesons (Christenson, Cronin, Fitch and Turlay (1964)), a few attempts have been undertaken to observe experimentally this phenomenon in different processes. However, that experiments have not been successful. At the present time, novel more precise experimental schemes are actively discussed: observation of the atom (Lamoreaux (1989)) and neutron (Forte (1983), Fedorov, Voronin and Lapin (1992)) electric dipole moment; the T(time)-violating atom's (molecule's) spin rotation in a laser wave and the T-violating refraction of a photon in a polarized atomic or molecular gas (Baryshevsky $(1993,1994)$ ).

\section{$1 \mathrm{P}$ and T-violating photon polarisation plane rota- tion.}

In accordance with Baryshevsky $(1993,1994)$ the $\mathrm{P}$ (parity)- and T-violating dielectric permittivity tensor $\varepsilon_{i k}$ (for case $\varepsilon_{i k}-\delta_{i k} \ll 1$ ) is given by

$$
\varepsilon_{i k}=\delta_{i k}+\chi_{i k}=\delta_{i k}+\frac{4 \pi \rho}{k^{2}} f_{i k}(0),
$$

where $\chi_{i k}$ is the polarizability tensor of the matter, $\rho$ is the number of atoms (molecules) per $\mathrm{cm}^{3}, k$ - the photon wave number. The quantity $f_{i k}(0)$ is the tensor part of the zero-angle amplitude of elastic coherent scattering of a photon by an atom (molecule) $f(0)=f_{i k}(0) e^{\prime^{*}} e_{k}$. Here $\vec{e}$ and $\vec{e}^{\prime}$ are the polarization vectors of initial and scattered photons. Indices $\mathrm{i}=1,2,3$ are refered to coordinates $\mathrm{x}, \mathrm{y}, \mathrm{z}$, respectively, repeated indices imply summations. At zero external electric and magnetic fields, the amplitude $f_{i k}(0)$ can be written as

$$
\begin{aligned}
f_{i k}(0)= & f_{i k}^{e v}(0)+f_{i k}^{P, T}(0)=f_{i k}^{e v}(0)+\frac{\omega^{2}}{c^{2}}\left[i \beta_{s}^{P} \varepsilon_{i k l} n_{l}-\beta_{v}^{P}(\langle\vec{J}\rangle \vec{n}) \delta_{i k}+\right. \\
& +i \beta_{t}^{P} \varepsilon_{i m l}\left\langle\hat{Q}_{m k}\right\rangle n_{l}+\frac{1}{2} \beta_{t}^{T}\left(\left\langle\hat{Q}_{i m}\right\rangle \varepsilon_{m l k} n_{l}+\left\langle\hat{Q}_{k m}\right\rangle \varepsilon_{m l i} n_{l}\right),
\end{aligned}
$$

where $f_{i k}^{e v}$ is the $\mathrm{P}, \mathrm{T}$ even (invariant) part of $f_{i k}(0), f_{i k}^{P, T}$ is the $\mathrm{P}, \mathrm{T}$-violating part of $f_{i k}(0), \beta_{s, v, t}^{P, T}$ is the scalar (vector, tensor) $\mathrm{P}, \mathrm{T}$ violating polarizability of an atom (molecule), $\varepsilon_{i k l}$ is the total antisymmetrical unit tensor of the rank three, $\vec{n}=\frac{\vec{k}}{k}$, $\langle\vec{J}\rangle=\operatorname{Sp\rho }_{J} \vec{J}, \rho_{J}$ is the spin density matrix of an atom (molecule) with the spin $\vec{J}$, 
$\left\langle Q_{i m}\right\rangle=S p \rho_{J} \hat{Q}_{i m}$. The second rank tensor $\hat{Q}_{i m}$ is given by

$$
\hat{Q}_{i m}=[2 J(2 J-1)]^{-1}\left\{\hat{J}_{i} \hat{J}_{m}+\hat{J}_{m} \hat{J}_{i}-\frac{2}{3} J(J+1) \delta_{i m}\right\}
$$

In view of $(1.1,1.2)$, the T-violating processes affect upon the dielectric permittivity $\varepsilon_{i k}$ and, as a result, upon the refraction index only in media with polarized atoms (molecules) of the spin equaled or larger than 1 . This part of $\varepsilon_{i k}$ is proportional to $\beta_{t}^{T}$. If the atoms' (molecules') spins are nonpolarized, only the P-violating term $f_{i k}(0)=\frac{\omega^{2}}{c^{2}} i \beta_{s}^{P} \varepsilon_{i k l} n_{l}$ exists. The term proportional to $\beta_{s}^{P}$ describes the $\mathrm{P}$-violating rotation of a light polarization plane in metallic vapours (Barkov and Zolotariov 1978, Bouchiat and Pottier 1986, Khriplovich 1991).

As it has been shown in (Baryshevsky 1993, 1994), when an atom interacts with two coherent electromagnetic waves, the energy of this interaction depends on the Tviolating scalar polarizability $\beta_{t}^{T}$. Interaction of an atom (molecule) with two waves can be considered as a process of rescattering of one wave into another and vice versa. Then, as it follows from an expression for the effective interaction energy, the amplitude $f(\vec{k}, \vec{k})$ of the photon scattering by an unpolarized atom (molecule) at a non-zero angle is given by (Baryshevsky 1994):

$$
f\left(\vec{k}^{\prime}, \vec{k}\right)=f_{i k} e_{i}^{e^{*}} e_{k}=\frac{\omega^{2}}{c^{2}}\left(\alpha_{s} \vec{e}^{*^{*}} \vec{e}+i \frac{1}{2} \beta_{s}^{P}\left(\vec{n}^{\prime}+\vec{n}\right)\left[\vec{e}^{*} \vec{e}\right]+\beta_{s}^{T}\left(\vec{n}^{\prime}-\vec{n}\right)\left[\vec{e}^{*} \vec{e}\right]\right),
$$

where $\vec{k}$ is the wave vector of a scattered photon, $\vec{n}^{\prime}=\frac{\vec{k}^{\prime}}{k}, \alpha_{s}$ is the scalar P,Tinvariant polarizability of an atom (molecule). Expression (1.3) holds true in the absence of .external electric and magnetic fields.

It should be emphasized that expression (1.3) for the elastic scattering amplitude can be derived from the general principles of symmetry. Indeed, there are four independent unit vectors: $\vec{\nu}_{1}=\frac{\vec{k}^{\prime}+\vec{k}}{\left|\vec{k}^{\prime}+\vec{k}\right|}, \vec{\nu}_{2}=\frac{\vec{k}^{\prime}-\vec{k}}{\left|\vec{k}^{\prime}-\vec{k}\right|}, \vec{e}$ and $\vec{e}^{\prime}$, which completely describe geometry of the elastic scattering process. The elastic scattering amplitude $f\left(\vec{k}^{\prime}, \vec{k}\right)$ depends on these vectors and therewith is a scalar. Obviously, one can compose three independent scalars from these vectors: $\vec{e}^{\prime} \vec{e}, \vec{\nu}_{1}\left[\vec{e}^{\prime^{\prime *}} \vec{e}\right], \vec{\nu}_{2}\left[\vec{e}^{\prime^{*}} \vec{e}\right]$. As a result, the scattering amplitude can be written as:

$$
f\left(\vec{k}^{\prime}, \vec{k}\right)=f_{s}\left(\vec{k}^{\prime}, \vec{k}\right) \vec{e}^{\prime^{*}} \vec{e}+i f_{s}^{P}(\vec{k}, \vec{k}) \vec{\nu}_{1}\left[\vec{e}^{\prime^{*}} \vec{e}\right]+f_{s}^{T}\left(\vec{k}^{\prime}, \vec{k}\right) \vec{\nu}_{2}\left[\vec{e}^{\prime^{*}} \vec{e}\right]
$$

where $f_{s}$ is the $\mathrm{P}$-,T- invariant scalar amplitude, $f_{s}^{P}$ is the $\mathrm{P}$-violating scalar amplitude, and $f_{s}^{T}$ is the $\mathrm{P}-, \mathrm{T}$ - violating scalar amplitude.

It can easily be found from $(1.3,1.4)$ that the term proportional to $\beta_{s}^{T}\left(f_{s}^{T}\right)$ vanishes in the case of forward scattering $\left(\vec{n}^{\prime} \rightarrow \vec{n}\right)$. Vice versa, in the case of back scattering $\left(\vec{n}^{\prime} \rightarrow-\vec{n}\right)$ the term proportional to $\beta_{s}^{P}\left(f_{s}^{P}\right)$ gets equal to zero. 
Thus, one can conclude that the T-violating interactions manifest themselves in the processes of scattering by atoms (molecules). However, the scattering processes are usually incoherent and their cross sections are too small to hope for observation of the T-violating effect. Another situation takes place for diffraction gratings in the vicinity of the Bragg resonance where the scattering process is coherent. As a result, the intensities of scattered waves strongly increase: for instance, in the Bragg (reflection) diffraction geometry the amplitude of the diffracted-reflected wave may reach the unity. It gives us an opportunity to study the T-violating scattering processes (Baryshevsky 1994).

In the present paper, equations describing the T-violating scattering by a diffraction grating have been obtained. It has been shown that the photon's refraction index in a non-center-symmetrical grating depends on the T-violating amplitude $f_{s}^{T}$. It can result in a new phenomenon: the T-violating rotation of the photon polarization plane. It has also been shown that the rotation angle rises sharply in the back-scattering diffraction geometry when the conditions of the photon resonance transmission are satisfied.

\section{The P-,T-violating electromagnetic waves diffraction by a diffrac- tion grating}

The phenomenon of $\mathrm{P}$-, T- invariant diffraction of electromagnetic waves by diffraction gratings has been studied in detail for a very wide range of wavelengths (see, for examples, Shih-Lin Chang (1984), Tamir (1988), Maksimenko and Slepyan (1997)). Accoding to these articles equations of the dynamic diffraction can be derived from the Maxwell's equations if the permittivity tensor $\varepsilon_{i k}(\vec{r}, \omega)$ of a spatially periodic grating is known.

To include the $\mathrm{P}, \mathrm{T}$ violating processes into the diffraction theory, let us consider the microscopic Maxwell equations:

$$
\begin{aligned}
\operatorname{curl} \vec{E} & =-\frac{1}{c} \frac{\partial \vec{B}}{\partial t} \quad, \quad \operatorname{curl} \vec{B}=\frac{1}{c} \frac{\partial \vec{E}}{\partial t}+\frac{4 \pi}{c} \vec{j} \\
\operatorname{div} \vec{E} & =4 \pi \rho \quad, \quad \operatorname{div} \vec{B}=0 \quad, \quad \frac{\partial \rho}{\partial t}+\operatorname{div} \vec{j}=0 .
\end{aligned}
$$

where $\vec{E}$ is the electric field strength and $\vec{B}$ is the magnetic field induction, $\rho$ and $\vec{j}$ are the microscopic densities of the electrical charge and the current induced by an electromagnetic wave, $c$ is the speed of light. The Fourier transformation of these equations (i.e. $\vec{E}(\vec{r}, t)=\frac{1}{2 \pi^{4}} \int \vec{E}(\vec{k}, \omega) e^{i \vec{k} \vec{r}} e^{-i \omega t} d^{3} k d \omega$ and so on) leads us to the equation for $\vec{E}(\vec{k}, \omega)$ as follows:

$$
\left(-k^{2}+\frac{\omega^{2}}{c^{2}}\right) \vec{E}(\vec{k}, \omega)=-\frac{4 \pi i \omega}{c^{2}}\left[\vec{j}(\vec{k}, \omega)-\frac{c^{2} k^{2}}{\omega^{2}} \vec{n}(\vec{n} \vec{j}(\vec{k}, \omega))\right]
$$

where $\vec{n}=\frac{\vec{k}}{k}$.

In linear approximation, the current $\vec{j}(\vec{r}, \omega)$ is coupled with $\vec{E}(\vec{r}, \omega)$ by the well-known dependence:

$$
j_{i}(\vec{r}, \omega)=\int d^{3} r^{\prime} \sigma_{i j}(\vec{r}, \vec{r}, \omega) E_{j}(\vec{r}, \omega)
$$


with $\sigma_{i j}(\vec{r}, \vec{r}, \omega)$ as the microscopic conductivity tensor being a sum of the conductivity tensors of the atoms (molecules) constituting the diffraction grating:

$$
\sigma_{i j}(\vec{r}, \vec{r}, \omega)=\sum_{A=1}^{N} \sigma_{i j}^{A}(\vec{r}, \vec{r}, \omega)
$$

Here $\sigma_{i j}^{A}$ is the conductivity tensor of the A-type scatterers. The summation is over all atoms (molecules) of the grating.

In a diffraction grating, the tensor $\sigma_{i j}(\vec{r}, \vec{r}, \omega)$ is a spatially periodic function. It allows one to derive the expansion of $j_{i}(\vec{k}, \omega)$ from $(1.7)$ as follows:

$$
j_{i}(\vec{k}, \omega)=\frac{1}{V_{0}} \sum_{\vec{\tau}} \sigma_{i j}^{c}(\vec{k}, \vec{k}-\vec{\tau}, \omega) E_{j}(\vec{k}-\vec{\tau}, \omega)
$$

where $\sigma_{i j}^{c}$ is the Fourier transform of the conductivity tensor of a grating's elementary cell, $\vec{\tau}$ is the reciprocal lattice vector of the diffraction grating. Using current representation (1.9), one can obtain a set of equations from (1.6):

$$
\left(-k^{2}+k_{0}^{2}\right) E_{i}(\vec{k}, \omega)=-\frac{\omega^{2}}{c^{2}} \sum_{\vec{\tau}} \hat{\chi}_{i j}(\vec{k}, \vec{k}-\vec{\tau}) E_{j}(\vec{k}-\vec{\tau})
$$

Tensor of the diffraction grating susceptibility is given by

$$
\hat{\chi}_{i j}(\vec{k}, \vec{k}-\vec{\tau})=\left(\delta_{i l}-n_{i} n_{l}\right) \chi_{l j}(\vec{k}, \vec{k}-\vec{\tau})
$$

with

$$
\chi_{l j}(\vec{k}, \vec{k}-\vec{\tau})=\frac{4 \pi i}{V_{0} \omega} \sigma_{l j}(\vec{k}, \vec{k}-\vec{\tau})=\frac{4 \pi c^{2}}{V_{0} \omega^{2}} F_{l j}(\vec{k}, \vec{k}-\vec{\tau}) .
$$

Here $F_{l j}(\vec{k}, \vec{k}-\vec{\tau})=\frac{i \omega}{c^{2}} \sigma_{l j}(\vec{k}, \vec{k}-\vec{\tau})$ is the amplitude of coherent elastic scattering of an electromagnetic wave by a grating elementary cell from a state with the wave vector $\vec{k}-\vec{\tau}$ to a state with the wave vector $\vec{k}$.

The amplitude $F_{l j}$ is obtained by summation of atomic (molecular) coherent elastic scatterig amplitudes over a grating's elementary cell:

$$
F_{l j}\left(\vec{k}^{\prime}=\vec{k}+\vec{\tau}, \vec{k}\right)=\left\langle\sum_{A=1}^{N_{c}} f_{l j}^{A}\left(\vec{k}^{\prime}=\vec{k}+\vec{\tau}, \vec{k}\right) e^{-i \vec{\tau} \vec{R}_{A}}\right\rangle,
$$

where $f_{l j}^{A}$ is the coherent elastic scattering amplitude by an A-type atom (molecule), $\vec{R}_{A}$ is the gravity center coordinate of the A-type atom (molecule), $N_{c}$ is the number of the atoms (molecules) in an elementary cell, angular brackets denote averaging over the coordinate distribution of scatterers in a grating's elementary cell.

The amplitude $f_{l j}$ has been given by equation $(1.4,1.3)$.

From (1.11), (1.12) and (1.4) one can obtaine an expression for the susceptibility $\chi_{l j}$ of the elementary cell of an optically isotropic material:

$$
\chi_{l j}(\vec{k}, \vec{k}-\vec{\tau})=\chi_{s \vec{\tau}} \delta_{l j}+i \chi_{s \vec{\tau}}^{P} \varepsilon_{l j f} \nu_{1 f}^{\vec{\tau}}+\chi_{s \vec{\tau}}^{T} \varepsilon_{l j f} \nu_{2 f}^{\vec{\tau}}
$$

where 


$$
\chi_{s \vec{\tau}}^{(P, T)}=\frac{4 \pi c^{2}}{V_{0} \omega^{2}}\left\langle\sum_{A=1}^{N_{c}} f_{s}^{A(P, T)}(\vec{k}, \vec{k}-\vec{\tau}) e^{-i \vec{\tau} \vec{R}_{A}}\right\rangle
$$

$\chi_{s \vec{\tau}}$ is the scalar P-, T- invariant susceptibility of an elementary cell, $\chi_{s \vec{\tau}}^{P}$ is the Pviolating, T- invariant susceptibility of the elementary cell, and $\chi_{s \vec{\tau}}^{T}$ is the $\mathrm{P}$ - and Tviolating susceptibility of the elementary cell,

$$
\overrightarrow{\nu_{1}}=\frac{2 \vec{k}-\vec{\tau}}{|2 \vec{k}-\vec{\tau}|} \quad, \quad \overrightarrow{\nu_{2}}=\frac{\vec{\tau}}{\tau}
$$

Then, using $(1.10,1.11,1.13)$ we can derive a set of equations describing the $\mathrm{P}$ and $\mathrm{T}$ violating interaction of an electromagnetic wave with a diffraction grating

$$
\begin{aligned}
\left(-\frac{k^{2}}{k_{0}^{2}}+1\right) E_{i}(\vec{k})= & -\left(\delta_{i j}-n_{i} n_{j}\right) \chi_{s 0} E_{j}(\vec{k})-i \chi_{s 0}^{P}\left(\delta_{i l}-n_{i} n_{l}\right) \varepsilon_{l j f} n_{f} E_{j}(\vec{k})- \\
& -\sum_{\vec{\tau} \neq 0}\left\{\left(\delta_{i j}-n_{i} n_{j}\right) \chi_{s \vec{\tau}} E_{j}(\vec{k}-\vec{\tau})+\right. \\
& +i \chi_{s \vec{\tau}}^{P}\left(\delta_{i l}-n_{i} n_{l}\right) \varepsilon_{l j f} \nu_{1 f}^{\vec{\tau}} E_{j}(\vec{k}-\vec{\tau})+ \\
& \left.+\chi_{s_{\vec{\tau}}}^{T}\left(\delta_{i l}-n_{i} n_{l}\right) \varepsilon_{l j f} \nu_{2 f}^{\vec{\tau}} E_{j}(\vec{k}-\vec{\tau})\right\}
\end{aligned}
$$

where $k_{0}=\frac{\omega}{c}$

Assuming the interaction to be $\mathrm{P}, \mathrm{T}$ invariant $\left(\chi_{s}^{P}=\chi_{s}^{T}=0\right)$, equations (1.14) reduce to the conventional set of equations of dynamic diffraction theory (Shih-Lin Chang (1984)).

\section{The phenomenon of T-violating rotation of the photon polariza- tion plane by a diffraction grating}

Let us suppose, first of all, the photon $\omega$ frequency and the wave vector $\vec{k}$ to be such that the Bragg diffraction conditions $\vec{k}=\vec{k} \pm \vec{\tau}$ and $|\vec{k}|=|\vec{k}|$ are not fulfilled exactly, and the inequality $\frac{k_{0}^{2}\left|\hat{\chi}_{l j}(\vec{k}, \vec{k}-\vec{\tau})\right|}{(\vec{k}-\vec{\tau})^{2}-k_{0}^{2}} \ll 1$ holds true. In this case, the diffracted wave amplitude is much less comparing with the transmitted one: $|\vec{E}(\vec{k}-\vec{\tau})| \ll|\vec{E}(\vec{k})|$, and the perturbation theory can be applied for the further analysis. As a result in the first approximation of the perturbation theory one can derive from (1.10) that

$$
\begin{aligned}
& E_{j}(\vec{k}-\vec{\tau}) \simeq \frac{k_{0}^{2}}{(\vec{k}-\vec{\tau})^{2}-k_{0}^{2}} \hat{\chi}_{j f}(\vec{k}-\vec{\tau}, \vec{k}) E_{f}(\vec{k})=\alpha_{\tau}^{-1} \hat{\chi}_{j f}(\vec{k}-\vec{\tau}, \vec{k}) E_{f}(\vec{k}), \\
& \text { where } \alpha_{\tau}=\frac{\vec{\tau}\left(\vec{\tau}-2 \vec{k}_{0}\right)}{k_{0}^{2}}, \vec{k}_{0}=\frac{\omega}{c} \vec{n},\left|\hat{\chi}_{i j}\right| \ll 1 .
\end{aligned}
$$


Substitution (1.15) into (1.10) results in the diffraction equations as follows

$$
\left(k^{2}-k_{0}^{2}\right) E_{i}(\vec{k}, \omega)-k_{0}^{2}\left[\hat{\chi}_{i f}(\vec{k}, \vec{k})+\sum_{\vec{\tau} \neq 0} \alpha_{\tau}^{-1} \hat{\chi}_{i j}(\vec{k}, \vec{k}-\vec{\tau}) \hat{\chi}_{j f}(\vec{k}-\vec{\tau}, \vec{k})\right] E_{f}(\vec{k}, \omega)=0
$$

which can be rewritten in more simple form

$$
\left(k^{2}-k_{0}^{2} \hat{\varepsilon}_{i f}(\vec{k}, \omega)\right) E_{f}(\vec{k}, \omega)=0
$$

by introducing the effective permittivity tensor

$$
\hat{\varepsilon}_{i f}(\vec{k}, \omega)=\delta_{i f}+\hat{\chi}_{j f}(\vec{k}, \vec{k}, \omega)+\sum_{\vec{\tau} \neq 0} \alpha_{\tau}^{-1} \hat{\chi}_{i j}(\vec{k}, \vec{k}-\vec{\tau}) \hat{\chi}_{j f}(\vec{k}-\vec{\tau}, \vec{k})
$$

One can see that even far away from the exact Bragg conditions, where the diffracted wave amplitudes are small, a spatially periodic isotropic medium manifests the optical anisotropy being characterized by the effective permittivity tensor $\hat{\varepsilon}_{i f}(\vec{k}, \omega)$.

Let a photon be incident on a grating normally to its reflection planes. In other words, let the photon wave vector $\vec{k}_{0}$ be antiparallel to the reciprocal lattice vector $\vec{\tau}$, i.e. $\vec{k}_{0} \uparrow \downarrow \vec{\tau}$. In this case, the back-scattering diffraction regime can be realized for photons with wave numbers defined by the relation $k \approx \frac{1}{2} \tau$. If, nevertheless, the inequality $\alpha_{\tau} \gg\left|\chi_{i j}(\vec{k}, \vec{k}-\vec{\tau})\right|$ holds true, we can use set of equations (1.16) in which there is only one term satisfying the conditions $\vec{\tau} \uparrow \downarrow \vec{k}$ and $\tau \simeq 2 k$.

Let the coordinate axis $z$ be parallel to $\vec{k}_{0}, \vec{\tau}$. In this case, the tensor $\hat{\chi}_{i j}$ has nonzero components at $i, j=1,2$ only. As a result, set of equations (1.17) can be rewritten in the form as follows:

$$
\left(k^{2}-k_{0}^{2} \varepsilon_{i j}(\vec{k}, \omega)\right) E_{j}(\vec{k}, \omega)=0
$$

with the permittivity tensor given by

$$
\begin{aligned}
\varepsilon_{i j}(\vec{k}, \omega)= & \varepsilon_{0} \delta_{i j}+i \chi_{s}^{P}(0) \varepsilon_{i j 3} n_{3}+ \\
& +\alpha_{\tau}^{-1}\left[\chi_{s}(\vec{\tau}) \chi_{s}^{T}(-\vec{\tau})-\chi_{s}(-\vec{\tau}) \chi_{s}^{T}(\vec{\tau})\right] \varepsilon_{i j 3} \nu_{23}^{\vec{\tau}}
\end{aligned}
$$

In the above equations we introduced the designations:

$$
\begin{aligned}
& \chi(\vec{\tau})=\chi(\vec{k}, \vec{k}-\vec{\tau}), \quad \chi(-\vec{\tau})=\chi(\vec{k}-\vec{\tau}, \vec{k}), \quad \varepsilon_{0}=1+\chi_{s}^{\text {eff }}, \\
& \chi_{s}^{\text {eff }}=\chi_{s}(0)-\alpha_{\tau}^{-1} \chi_{s}(\vec{\tau}) \chi_{s}(-\vec{\tau}) \quad, \quad \vec{n}=\frac{\vec{k}}{k} \quad, \quad \vec{\nu}_{2}^{\tau}=\frac{\vec{\tau}}{\tau}
\end{aligned}
$$

The term proportional $t_{0} \chi_{s}^{P}(0)$ describes the P-violating and T-invariant rotation of the light polarization plane about the direction $\vec{n}$. This term does not depend on a structure of the diffraction grating and exists for any ordinary spatially isotropic media. Unlike to that, the term proportional to $\chi_{s}^{T}$ is T-violating and depends on the grating's structure. The term proportional $\chi_{s}^{T}$ looks like the term proportional to $\chi_{s}^{P}$ and is responsible for the polarization plane rotation about $\vec{\nu}_{2}^{\tau}$. 
It is known that the phenomenon of the polarization plane rotation arises when rightand left-circularly polarized photons have different indices of refraction in a medium $n_{+}$ and $n_{-}$, respectively. It means that the tensor $\varepsilon_{i j}$ is diagonal for a given circular polarization and, concequently, the set of equations (1.19) is split into two independent equations. Really, let us write (1.19) in the vector notation:

$$
\left(k^{2}-k_{0}^{2} \varepsilon_{0}\right) \vec{E}-i k_{0}^{2} \chi_{s}^{P}(0)[\vec{E} \vec{n}]-k_{0}^{2} \alpha_{\tau}^{-1}\left[\chi_{s}(\vec{\tau}) \chi_{s}^{T}(-\vec{\tau})-\chi_{s}(-\vec{\tau}) \chi_{s}^{T}(\vec{\tau})\right]\left[\vec{E} \vec{\nu}_{2}^{\tau}\right]
$$

and let $\vec{e}_{1}$ be the unit polarization vector of a linearly polarized photon; $\vec{e}_{2}=\left[\vec{n} \vec{e}_{1}\right]$, $\vec{e}_{1} \perp \vec{e}_{2} \perp \vec{n}$. Then, the unit vectors corresponding to the circular polarizations are as follows $\vec{e}_{ \pm}=\frac{\vec{e}_{1} \pm i \vec{e}_{2}}{\sqrt{2}}$. For the right $\left(\vec{e}_{+}\right)$, left $\left(\vec{e}_{-}\right)$circularly polarized photons, the field $\vec{E}$ can be represented by $\vec{E}=c_{( \pm)} \vec{e}_{( \pm)}$. As a result, it follows from $(1.19,1.21)$ that:

$$
\left(k^{2}-k_{0}^{2} \varepsilon_{0}\right) c_{ \pm} \pm k_{0}^{2} \chi_{s}^{P}(0) c_{ \pm} \pm i k_{0}^{2} \alpha_{\tau}^{-1}\left[\chi_{s}(\vec{\tau}) \chi_{s}^{T}(-\vec{\tau})-\chi_{s}(-\vec{\tau}) \chi_{s}^{T}(\vec{\tau})\right] c_{ \pm}=0
$$

The corresponding refractive indices are obtained from (1.20)

$$
n_{ \pm}^{2}=\frac{k^{2}}{k_{0}^{2}}=\varepsilon_{0} \mp \chi_{s}^{P}(0) \mp i \alpha_{\tau}^{-1}\left[\chi_{s}(\vec{\tau}) \chi_{s}^{T}(-\vec{\tau})-\chi_{s}(-\vec{\tau}) \chi_{s}^{T}(\vec{\tau})\right]
$$

The angle of the photon polarization plane rotation is defined by :

$$
\vartheta=k_{0} R e\left(n_{+}-n_{-}\right) L_{p h}
$$

where $L_{p h}$ is the photon propagation length in the medium, Ren $n_{ \pm}$is the real part of $n_{ \pm}$. Then, the expression for $\vartheta$ can easily be derived from $(1.24,1.23)$

$$
\begin{aligned}
\vartheta= & k_{0} \operatorname{Re} \frac{n_{+}^{2}-n_{-}^{2}}{n_{+}+n_{-}} L \simeq-k_{0} \operatorname{Re} \chi_{s}^{P}(0) L- \\
& -k_{0} \operatorname{Rei\alpha }_{\tau}^{-1}\left[\chi_{s}(\vec{\tau}) \chi_{s}^{T}(-\vec{\tau})-\chi_{s}(-\vec{\tau}) \chi_{s}^{T}(\vec{\tau})\right] L
\end{aligned}
$$

One can conclude, thus, that the T-violating interaction results in the phenomenon of the T-violating rotation of the photon polarization plane. The effect manifests itself when the condition $\operatorname{Re} i\left[\chi_{s}(\vec{\tau}) \chi_{s}^{T}(-\vec{\tau})-\chi_{s}(-\vec{\tau}) \chi_{s}^{T}(\vec{\tau})\right] \neq 0$ holds true.

It follows from (1.13) that the susceptibilities $\chi_{s}^{P, T}(\vec{\tau})$ can be presented as

$$
\begin{aligned}
\chi_{s}^{P, T}(\vec{\tau})= & \chi_{1 s}^{P, T}(\vec{\tau})-\chi_{2 s}^{P, T}(\vec{\tau})=\left\langle\sum_{A=1}^{N_{c}} f_{s}^{A(P, T)}(\vec{k}, \vec{k}-\vec{\tau}) \cos \vec{\tau} \vec{R}_{A}\right\rangle- \\
& -i\left\langle\sum_{A=1}^{N_{c}} f_{s}^{A(P, T)}(\vec{k}, \vec{k}-\vec{\tau}) \sin \vec{\tau} \vec{R}_{A}\right\rangle
\end{aligned}
$$

where

$$
\chi_{1 s}^{P, T}(\vec{\tau})=\chi_{1 s}^{P, T}(-\vec{\tau}) \quad \chi_{2 s}^{P, T}(\vec{\tau})=-\chi_{2 s}^{P, T}(-\vec{\tau})
$$


In view of $(1.26,1.27)$ we can rewrite $(1.25)$ as:

$$
\vartheta=-k_{0} \operatorname{Re} \chi_{s}^{P}(0) L+2 k_{0} \alpha_{\tau}^{-1} \operatorname{Re}\left[\chi_{1 s}(\vec{\tau}) \chi_{2 s}^{T}(\vec{\tau})-\chi_{2 s}(\vec{\tau}) \chi_{1 s}^{T}(\vec{\tau})\right] L
$$

So, the T-violating rotation arises in the case of nonzero odd part of the suscetibility: $\chi_{2}(\vec{\tau}) \neq 0$. Such a situation is possible if an elementary cell of the diffraction grating does not posses the center of symmetry.

In accordance with (1.28), the angle of the T-violating rotation grows at $\alpha_{\tau} \rightarrow 0$. .However, the condition $\alpha_{\tau}\left|\chi_{s}(\vec{\tau})\right| \ll 1$ violates at $\alpha_{\tau}^{-1} \rightarrow 0$, where the amplitude of diffracted and transmitted waves are comparable: $E(\vec{k}-\vec{\tau}) \simeq E(\vec{k})$ and, consequently, the perturbation theory gets unapplicable. A rigorous dynamical diffraction theory must be applied in this case.

\section{The T-violating polarization plane rotation in the Bragg diffrac- tion scheme}

Let the Bragg condition is fulfilled for the only diffracted wave and is violated for all other possible ones. It allows us to restrict ourselves to the two-wave approximation of the dynamical diffraction theory (Shih-Lin Chang (1984)). In that case, set of equations

(1.14) reduces to two coupled equations, which for the back-scattering diffraction scheme $\left(\vec{k}_{0} \| \vec{\tau}\right)$ take the form as follows:

$$
\begin{aligned}
& \left(\frac{k^{2}}{k_{0}^{2}}-1\right) E_{j}(\vec{k})=\chi_{s}(0) E_{j}(\vec{k})+i \chi_{s}^{P}(0) \varepsilon_{j m f} E_{m}(\vec{k}) n_{f}+ \\
& +\chi_{s}(\vec{\tau}) E_{j}(\vec{k}-\vec{\tau})+\chi_{s}^{T}(\vec{\tau}) \varepsilon_{j m f} E_{m}(\vec{k}-\vec{\tau}) \nu_{2 f}^{\vec{\tau}}, \\
& \left(\frac{(\vec{k}-\vec{\tau})^{2}}{k_{0}^{2}}-1\right) E_{j}(\vec{k}-\vec{\tau})=\chi_{s}(0) E_{j}(\vec{k}-\vec{\tau})+ \\
& i \chi_{s}^{P}(0) \varepsilon_{j m f} n_{f}(\vec{k}-\vec{\tau}) E_{m}(\vec{k}-\vec{\tau})+ \\
& +\chi_{s}(-\vec{\tau}) E_{j}(\vec{k})+\chi_{s}^{T}(-\vec{\tau}) \varepsilon_{j m f} \nu_{2 f}^{-\vec{\tau}} E_{m}(\vec{k}), \\
& \vec{n}(\vec{k}-\vec{\tau})=\frac{\vec{k}-\vec{\tau}}{|\vec{k}-\vec{\tau}|} .
\end{aligned}
$$

Based on the above consideration, we can conclude that set of equations (1.29) can be diogonalized for the photon of a given circular polarization. Let the right-circularly polarized photon $\left(\vec{e}_{+}\right)$be incident on the diffraction grating. The diffraction process, as it follows from (29), results in the appearance of a back-scattered photon with the left circular polarization $\left(\overrightarrow{e_{-}^{\tau}}\right)$. This is because the momentum of the back-scattered photon $\vec{k}=\vec{k}-\vec{\tau}$ is antiparallel to the momentum $\vec{k}$ of the incident one. It is obvious that the left-circularly polarized photon will produce a right-circularly polarized back-scattered one. 
Thus, for circularly polarized photons set of vector equations (1.29) can be split into two independent sets of scalar equations:

$$
\begin{aligned}
\left(\frac{k^{2}}{k_{0}^{2}}-1\right) C_{ \pm}(\vec{k})= & \left(\chi_{s}(0) \mp \chi_{s}^{P}(0)\right) C_{ \pm}(\vec{k})+ \\
& +\left(\chi_{s}(\tau) \mp i \chi_{s}^{T}(\tau)\right) C_{\mp}(\vec{k}-\vec{\tau}) \\
\left(\frac{(\vec{k}-\vec{\tau})}{k_{0}^{2}}-1\right) C_{\mp}(\vec{k}-\vec{\tau})= & \left(\chi_{s}(0) \pm \chi_{s}^{P}(0)\right) C_{\mp}(\vec{k}-\vec{\tau})+ \\
& +\left(\chi_{s}(-\vec{\tau}) \pm i \chi_{s}^{T}(-\vec{\tau})\right) C_{ \pm}(\vec{k})
\end{aligned}
$$

Note that equations (1.30) are identical in form to conventional equations of the twowave dynamical diffraction (Shih-Lin Chang (1984)):

$$
\begin{aligned}
& \left(\frac{k^{2}}{k_{0}^{2}}-1\right) c_{0}=\chi_{0} c_{0}+\chi_{\tau} c_{\tau} \\
& \left(\frac{k_{\tau}^{2}}{k_{0}^{2}}-1\right) c_{\tau}=\chi_{0} c_{\tau}+\chi_{-\tau} c_{0}
\end{aligned}
$$

It allows us to write down a solution immediately, without deriving (see, for example, (Shih-Lin Chang (1984))). As a result, the amplitude of the transmitted electromagnetic wave at the output is given by

$$
\begin{gathered}
\vec{E}_{ \pm}=\vec{e}_{ \pm} C_{ \pm}(L) e^{i \vec{k}_{0} \vec{r}} \\
C_{ \pm}(L)=2\left(\varepsilon_{1}^{ \pm}-\varepsilon_{2}^{ \pm}\right) e^{i \frac{1}{2} k_{0} \varepsilon^{ \pm} L}\left[\left(2 \varepsilon_{1}^{ \pm}-\chi_{0}^{ \pm}\right) e^{i \frac{1}{2} k_{0}\left(\varepsilon_{1}^{ \pm}-\varepsilon_{2}^{ \pm}\right) L}-\right. \\
\left.-\left(2 \varepsilon_{2}^{ \pm}-\chi_{0}^{ \pm}\right) e^{-i \frac{1}{2}\left(\varepsilon_{1}^{ \pm}-\varepsilon_{2}^{ \pm}\right) L}\right]^{-1},
\end{gathered}
$$

where $\mathrm{L}$ is the thickness of the diffraction grating,

$$
\begin{gathered}
\chi_{0}^{ \pm}=\chi_{s}(0) \mp \chi_{s}^{P}(0), \\
\varepsilon_{1}^{ \pm}=\frac{1}{4}\left[\mp 2 \chi_{s}^{P}(0)+\alpha\right]+\frac{1}{4}\left\{\left(2 \chi_{s}(0)-\alpha\right)^{2}-4\left(\chi_{1 s}^{2}(\vec{\tau})+\chi_{2 s}^{2}(\vec{\tau})\right) \pm\right. \\
\\
\left. \pm 8\left[\chi_{1 s}(\vec{\tau}) \chi_{2 s}^{T}(\vec{\tau})-\chi_{2 s}(\vec{\tau}) \chi_{1 s}^{T}(\vec{\tau})\right]\right\}^{\frac{1}{2}}, \\
\varepsilon_{2}^{ \pm}=\frac{1}{4}\left[\mp 2 \chi_{s}^{P}(0)+\alpha\right]-\frac{1}{4}\left\{\left(2 \chi_{s}(0)-\alpha\right)^{2}-4\left(\chi_{1 s}^{2}(\vec{\tau})+\chi_{2 s}^{2}(\vec{\tau})\right) \pm\right. \\
\left. \pm 8\left[\chi_{1 s}(\vec{\tau}) \chi_{2 s}^{T}(\vec{\tau})-\chi_{2 s}(\vec{\tau}) \chi_{1 s}^{T}(\vec{\tau})\right]\right\}^{\frac{1}{2}},
\end{gathered}
$$




$$
\begin{aligned}
& \alpha \equiv \alpha_{\tau}, \\
& \varepsilon^{ \pm}=\varepsilon_{1}^{ \pm}+\varepsilon_{2}^{ \pm}=\mp \chi_{s}^{P}(0)+\frac{\alpha}{2} \\
& \varepsilon_{1}^{ \pm}-\varepsilon_{2}^{ \pm}= \frac{1}{2}\left\{\left(2 \chi_{s}(0)-\alpha\right)^{2}-4\left(\chi_{1 s}^{2}(\vec{\tau})+\chi_{2 s}^{2}(\vec{\tau})\right) \pm\right. \\
&\left. \pm 8\left[\chi_{1 s}(\vec{\tau}) \chi_{2 s}^{T}(\vec{\tau})-\chi_{2 s}(\vec{\tau}) \chi_{1 s}^{T}(\vec{\tau})\right]\right\}^{\frac{1}{2}}
\end{aligned}
$$

Consider now the diffraction of a photon with linear polarization $\vec{e}_{1}$ being the superposition of two opposite circular polarizations. In this case

$$
\vec{E}(\vec{r})=\vec{e}_{1} e^{i \vec{k}_{0} \vec{r}}=\frac{\vec{e}_{+}+\vec{e}_{-}}{\sqrt{2}} e^{i \vec{k}_{0} \vec{r}}
$$

and the amplitude of the transmitted wave $\vec{E}^{\prime}(r)$ can be presented by the superposition

$$
\begin{aligned}
\vec{E}^{\prime}(\vec{r}) & =\left(\frac{1}{\sqrt{2}} \vec{e}_{+} C_{+}(L)+\frac{1}{\sqrt{2}} \vec{e}_{-} C_{-}(L)\right) e^{i \vec{k}_{0} \vec{r}}= \\
& =\left(\frac{\vec{e}_{1}+i \vec{e}_{2}}{2} C_{+}(L)+\frac{\vec{e}_{1}-i \vec{e}_{2}}{2} C_{-}(L)\right) e^{i \vec{k}_{0} \vec{r}}= \\
& =\left(\frac{C_{+}+C_{-}}{2} \vec{e}_{1}+i \frac{C_{+}-C_{-}}{2} \vec{e}_{2}\right) e^{i \vec{k}_{0} \vec{r}} .
\end{aligned}
$$

In the case under consideration $c_{+} \neq c_{-}$.As follows from (1.38), this results in changing of the photon polarization at the output.

Let us analyze expression (1.32) for the transmitted wave amplitude more attentively. According to (1.32), the amplitude oscillates as a function of $\alpha$, i.e., as a function of the wavelength, with maximums in points defined by the condition $k_{0} R e\left(\varepsilon_{1}^{ \pm}-\varepsilon_{2}^{ \pm}\right) L=2 \pi m$ or $\operatorname{Re}\left(\varepsilon_{1}^{ \pm}-\varepsilon_{2}^{ \pm}\right)=\frac{2 \pi m}{k_{0} L}$ with $m$ as an integer.

Note first of all that the condition $m=0$ dictates at $\varepsilon_{1}^{ \pm}-\varepsilon_{2}^{ \pm} \rightarrow 0$ the limit transition

$$
\left\{\left(2 \chi_{s}(0)-\alpha\right)^{2}-4\left(\chi_{1 s}^{2}(\vec{\tau})+\chi_{2 s}^{2}(\vec{\tau})\right) \pm 8\left[\chi_{1 s}(\vec{\tau}) \chi_{2 s}^{T}(\vec{\tau})-\chi_{2 s}(\vec{\tau}) \chi_{1 s}^{T}(\vec{\tau})\right]\right\}^{\frac{1}{2}} \rightarrow 0
$$

which determines the thresholds of the total Bragg reflection band where there is a quickly damped inhomogeneous wave inside the diffraction grating. As a result, the transmitted wave amplitude is small.

Let now $m \neq 0$. As we know, the T-violating interactions are very small: $\chi_{s 1,2}^{T} \ll \chi_{s 1,2}$. It allows one to expand the square root into the Teylor series truncated beyond the second term:

$$
\varepsilon_{1}^{ \pm}-\varepsilon_{2}^{ \pm} \simeq\left(\varepsilon_{1}-\varepsilon_{2}\right)_{e v} \pm \frac{\chi_{1 s}(\vec{\tau}) \chi_{2 s}^{T}(\vec{\tau})-\chi_{2 s}(\vec{\tau}) \chi_{1 s}^{T}(\vec{\tau})}{\left(\varepsilon_{1}-\varepsilon_{2}\right)_{e v}}
$$


where $\left(\varepsilon_{1}-\varepsilon_{2}\right)_{e v}=\frac{1}{2}\left\{\left(2 \chi_{s}(0)-\alpha\right)^{2}-4\left(\chi_{1 s}^{2}(\vec{\tau})+\chi_{2 s}^{2}(\vec{\tau})\right)\right\}^{\frac{1}{2}}$.

Let $R e \chi_{s 1,2} \gg \operatorname{Im} \chi_{s 1,2}$, i.e., the absorption is assumed to be sufficiently small to satisfy the condition $k_{0} \operatorname{Im}\left(\varepsilon_{1}-\varepsilon_{2}\right)_{e v} L \ll 1$ which admits the consideration of the diffraction grating as an optically transparent medium with $\left(\varepsilon_{1}-\varepsilon_{2}\right)_{e v}$ and $\chi_{s}$ as real functions. Let now the condition $k_{0}\left(\varepsilon_{1}-\varepsilon_{2}\right)_{e v} L=2 \pi m$ be fulfilled at $m \neq 0$. This condition defines the resonance transmission in the grating and allows us to rewrite formula (1.40) in the form as follows

$$
\varepsilon_{1}^{ \pm}-\varepsilon_{2}^{ \pm}=\frac{2 \pi m}{k_{0} L} \pm \frac{k_{0}\left[\chi_{1 s}(\vec{\tau}) \chi_{2 s}^{T}(\vec{\tau})-\chi_{2 s}(\vec{\tau}) \chi_{1 s}^{T}(\vec{\tau})\right] L}{2 \pi m}
$$

By substituting (1.41) into $(1.33,1.32)$ one can express $\vec{E}_{ \pm}$by

$$
\vec{E}_{ \pm}=\vec{e}_{ \pm}(-1)^{m}\left(1-i \frac{k_{0}\left(\alpha_{1,2}-2 \chi_{s}(0)\right) L}{8 \pi m} k_{0} \Delta^{ \pm} L\right) e^{i k_{0} \frac{1}{2} \varepsilon^{ \pm}\left(\alpha_{1,2}\right) L}
$$

where

$$
\begin{gathered}
\alpha_{1,2}=2 \chi_{s}(0) \pm \sqrt{4\left(\chi_{1 s}^{2}+\chi_{2 s}^{2}\right)+\left(\frac{4 \pi m}{k_{0} L}\right)^{2}} \\
\varepsilon^{ \pm}\left(\alpha_{1,2}\right)=\mp \chi_{s}^{P}(0)+\frac{1}{2} \alpha_{1,2}
\end{gathered}
$$

In view of that the second term in (1.42) is small and expression (1.42) can be written as

$$
\vec{E}_{ \pm}=\vec{e}_{ \pm}(-1)^{m} e^{i \varphi \pm}
$$

where the phase terms are given by

$$
\varphi_{ \pm}=k_{0}\left[\frac{1}{2} \varepsilon^{ \pm}\left(\alpha_{1,2}\right)-\frac{k_{0}\left(\alpha_{1,2}-2 \chi_{s}(0)\right) L}{8 \pi m} \Delta^{ \pm}\right] L
$$

Using this equation one can find the angle of the polarization plane rotation:

$$
\vartheta=\operatorname{Re}\left(\varphi_{+}-\varphi_{-}\right)=\vartheta^{P}+\vartheta_{1,2}^{T}
$$

where the first term in the right-hand part defines the $\mathrm{P}$-violating T-invariant rotation angle:

$$
\vartheta^{P}=-k_{0} \operatorname{Re} \chi_{s}^{P}(0) L
$$

and the second one corresponds to the T-violating rotation:

$$
\begin{aligned}
\vartheta_{1,2}^{T}\left(\alpha_{1,2}\right)= & \mp \frac{k_{0}^{3} L^{3}}{8 \pi^{2} m^{2}} \sqrt{4\left(\chi_{1 s}^{2}+\chi_{2 s}^{2}\right)+\left(\frac{4 \pi m}{k_{0} L}\right)^{2}} \times \\
& \times\left[\chi_{1 s}(\vec{\tau}) \operatorname{Re} \chi_{2 s}^{T}(\vec{\tau})-\chi_{2 s}(\vec{\tau}) \operatorname{Re} \chi_{1 s}^{T}(\vec{\tau})\right],
\end{aligned}
$$

the $\operatorname{sign}(-)$ is for $\alpha_{1}$, the $\operatorname{sign}(+)$ is for $\alpha_{2}$. 
The imaginary part of the T-violating polarizability $\operatorname{Im} \chi_{s 1,2}^{T}$ is responsible for the T-violating circular dichroism. Due to that process, a linearly polarized photon gets a circular polarization at the diffraction grating's output. The degree of the circular polarization of the photon is determined from the relation:

$$
\begin{aligned}
\delta_{1,2}= & \frac{\left|\vec{E}_{+}\right|^{2}-\left|\vec{E}_{-}\right|^{2}}{\left|\vec{E}_{+}\right|^{2}+\left|\vec{E}_{-}\right|^{2}} \simeq \operatorname{Im} \varphi_{-}-\operatorname{Im} \varphi_{+}=k_{0} \operatorname{Im} \chi_{s}^{P}(0) L \pm \\
& \pm \frac{k_{0}^{3} L^{3}}{8 \pi^{2} m^{2}} \sqrt{4\left(\chi_{1 s}^{2}+\chi_{2 s}^{2}\right)+\left(\frac{4 \pi m}{k_{0} L}\right)^{2}}\left[\chi_{1 s}(\vec{\tau}) \operatorname{Im} \chi_{2 s}^{T}(\vec{\tau})-\chi_{2 s}(\vec{\tau}) \operatorname{Im} \chi_{1 s}^{T}(\vec{\tau})\right]
\end{aligned}
$$

It should be pointed out that the resonance transmission condition is satisfied at a given $\mathrm{m}$ for two different values of $\alpha$. This is because there is a possibility to approach to the Brilluan (the total Bragg reflection) bandgap both from high and low frequencies. The T-violating parts of the rotation angle are opposite in sign for $\alpha_{1}$ and for $\alpha_{2}$. It gives the addition opportunity to distinguish the T-violating rotation from the $\mathrm{P}$-violating $\mathrm{T}$ invariant rotation. Indeed, the P-violating rotation does not depend on the back Bragg diffraction in the general case because the P-violating scattering amplitude equals zero for back scattering (see (1.32-1.35).

In accordance with $(1.47,1.48)$ the T-violating rotation and dichroism grow sharply in the vicinity of the resonance Bragg transmission. At the first glance, one could expect for $\vartheta^{T}$ the dependence $\vartheta^{T} \sim k_{0} \operatorname{Re} \chi_{s 1,2}^{T}(\vec{\tau}) L$ (see (1.25)). However, in the vicinity of resonance, the rotation angle $\vartheta^{T}$ turns out to be multiplied by the factor $A=$ $\left(8 \pi^{2} m^{2}\right)^{-1} k_{0} \sqrt{4\left(\chi_{1 s}^{2}+\chi_{2 s}^{2}\right)+\left(\frac{4 \pi m}{k_{0} L}\right)^{2}} L k_{0} \chi_{s 1,2} L$ which provides the above mentioned growth (for example, $A \sim 10^{5}$ at $\chi_{s} \simeq 10^{-1}, k_{0} \simeq 10^{4} \div 10^{5} \mathrm{~cm}^{-1}, L=1 \mathrm{~cm}, m=1$ ).

There are different types of diffraction gratings destined for use in optical and more longwave ranges. However, it should be noted that the successful observation of the Pviolating rotation has been performed by means of studying of light transmission through gas targets. There are a lot of theoretical calculations for atoms of such gases: see, for example, (Khriplovich (1991)) for $B i, T l, P b, D y$. From that point of view, it would be preferable to use gases for studying of the T-violating phenomena of polarization plane rotation and dichroism applying the experience accumulated earlier. At the first glance, there is a serious problem how to create such a diffraction grating in a gas. Nevertheless, the problem can be solved if we make use some well-known results of the electromagnetic theory of waveguides (Jackson (1962), Tamir (1988), Maksimenko and Slepyan (1997)). According to this theory, there is a correspondence between wave processes in waveguides with periodically modulated boundaries and homogeneous filling and such processes in regular waveguides filled by a periodic and, generally, anisotropic medium . Let us consider a regular waveguide constituted by two plane-parallel surfaces; for example, two metallic or dielectric mirrors. Let us then place a plane diffraction grating on the surface of the mirror and fill the waveguide by the studied gas. Because of the above stated correspondence, such a system is equivalent to a regular plane waveguide filled by a gas with a spatially periodic permittivity tensor. The permittivity modulation period is therewith equal to the grating period. As the chosen plane grating has an asymmetric profile, the corresponding virtual volume grating of the permittivity turns 
out to be noncentrosymmetrical and, thus, satisfies to the above imposed requirements for displaying of the T-violating phenomena.

Let $\hat{\varepsilon}_{i j}(r, \omega)=1+\hat{\chi}_{i j}(x, z)$ be the permittivity of the waveguide being considered with as a periodic function with respect to $z$. As it has been stated above, such a waveguide can be modeled by the waveguide with the effective permittivity $\hat{\varepsilon}_{\text {eff }}(z, \omega)=1+\hat{\chi}_{\text {eff }}(z)$ which is a periodic function of $z$ and is independent on $x$. We can show it mathematically starting with the Maxwell equations

$$
\text { curlcurl } \vec{E}(\vec{r}, \omega)-\frac{\omega^{2}}{c^{2}} \hat{\varepsilon}(r, \omega) \vec{E}(\vec{r}, \omega)=0
$$

Let an electromagnetic wave propagate in the plane regular waveguide. In this case, the motion along the $z$-axis is free and, thus, it can be described by a plane wave $e^{i k z}$. It results in a set of equations for determining of the stationary states of the waveguide:

$$
\frac{d^{2} \vec{E}_{n \alpha}(x)}{d x^{2}}+\kappa_{n}^{2} \vec{E}_{n \alpha}(x)=0
$$

where $\kappa_{n}=\frac{2 \pi n}{d}, \alpha$ notes the polarization state of the wave.

Then, the field $\vec{E}(\vec{r}, \omega)$ in the waveguide with the diffraction grating can be presented by the expansion as follows

$$
\vec{E}(\vec{r}, \omega)=\sum_{n} c_{n \alpha}(z) \vec{E}_{n \alpha}(x)
$$

Substitution of this expansion into (1.50) yields

$$
\frac{\partial^{2}}{\partial z^{2}} c_{n \alpha}(z)+\left(\frac{\omega^{2}}{c^{2}}-\kappa_{n}^{2}\right) c_{n \alpha}(z)+\frac{\omega^{2}}{c^{2}} \sum_{\tau \alpha} \hat{\chi}_{\tau \alpha \alpha^{\prime}}^{n n^{\prime}} e^{-i \tau z} c_{a^{\prime}}(z)=0
$$

where $\hat{\chi}_{\tau \alpha \alpha^{\prime}}^{n n^{\prime}}=\int\left\langle E_{n \alpha}(x)\left|\chi_{\tau}(x)\right| E_{n^{\prime} \alpha^{\prime}}(x)\right\rangle d x, \chi_{\tau}=\frac{1}{a} \int_{0}^{a} \chi(z) e^{i \tau z} d z, a$ - is the period of the plane grating.

Assuming the inequality $\frac{\chi^{n n^{\prime}} \frac{\omega^{2}}{c^{2}}}{\kappa_{n}^{\prime 2}-\kappa_{n}^{2}} \ll 1, \chi_{n n} \ll 1$ to be valid, we can restrict ourselves to a single mode approximation, which allows us to rewrite (1.50) as

$$
\frac{\partial^{2}}{\partial z^{2}} c_{n \alpha}(z)+\left(\frac{\omega^{2}}{c^{2}}-\kappa_{n}^{2}\right) c_{n \alpha}(z)+\frac{\omega^{2}}{c^{2}} \hat{\chi}_{\alpha \alpha^{\prime}}(z) c_{\alpha^{\prime}}(z)=0
$$

where $\hat{\chi}_{\alpha \alpha^{\prime}}(z)=\sum_{\tau} \hat{\chi}_{\tau \alpha \alpha^{\prime}}^{n n^{\prime}} e^{-i \tau z}$.

Equations (1.52) are identical with those (see (1.10)) describing the electric field in a volume diffraction grating. So, the goal formulated above has been achieved: we have obtained a volume diffraction grating.

Let us remind (see (1.18)) that the diffraction grating manifests the optical anisotropy (birefringens if the matter of grating possesses optical isotropy). The anisotropy makes difficult to observe the T-violating photon polarization plane rotation. Luckely, however, if the matter of the waveguide or diffraction grating is a dielectric one can change the optical anisotropy characteristics and even decrease the anysotropy sharply by the electric field due to the Kerr or Pockels effect. 
Now, let us estimate the effect of the T-violation. To do that we must determine, in accordance with formula (1.47) for $\vartheta^{T}$, the T-violating susceptibility $\chi_{s 1,2}^{T}$, which, in view of $(1.13,1.3,1.4)$, is proportional to the T-violating atomic polarizability $\beta_{s}^{T}$. The estimate carried out by (Baryshevsky (1993,1994), Khriplovich (1991)) gives $\beta_{s}^{T} \sim 10^{-3} \div 10^{-4} \beta_{s}^{P}$, where $\beta_{s}^{P}$ is the $\mathrm{P}$-violating T-invariant scalar polarizability. The polarizability $\beta_{s}^{P}$ was studied both theoretically and experimentally (Khriplovich (1991)). Particularly, the theory gives $\beta_{s}^{P} \cong 10^{-30} \mathrm{~cm}^{3}$ for atoms analogous to $\mathrm{Bi}, \mathrm{Tl}, \mathrm{Pb}$. It yields the estimate $\cong 10^{-33} \div 10^{-34} \mathrm{~cm}^{3}$ for the T-violating atomic polarizability. The polarizabelity $\beta_{s}^{P}$ causes the P-violating rotation of the polarization plane by the angle $\vartheta^{P}=k \operatorname{Re} \chi_{s}^{P}(0) L \cong 10^{-7}$ $\mathrm{rad} / \mathrm{cm} \times \mathrm{L}$ for the gas density $\rho=10^{16} \div 10^{17}$. As a result, in our case the parameter $\varphi=k \chi_{s}^{T}(\tau) L$ turns out to be $\varphi \cong 10^{-10} \div 10^{-11} \mathrm{rad} / \mathrm{cm} \times \mathrm{L}$ and can be even less by the factor $\mathrm{h} / \mathrm{d}$, where $\mathrm{h}$ is the corrugation amplitude of the diffraction grating while $\mathrm{d}$ is the distance between waveguide's mirrors. Assuming this factor to be $\sim 10^{-1}$, we shall find $\varphi \cong 10^{-11} \div 10^{-12} \mathrm{rad} / \mathrm{cm} \times \mathrm{L}$. Thus, the final estimate of the T-violating rotation angle $\vartheta^{T}$ is

$$
\vartheta^{T} \cong 10^{-11} \div 10^{-12} k_{0}{ }^{2} \chi_{s}^{2}(\tau) L^{3}
$$

In real situation the susceptibility of a grating $\chi_{s}(\tau)$ may exceed the unity. However, our analysis has been performed under the assumption $\chi_{s} \ll 1$. If, for example, we take $\chi_{s}=10^{-1}, k_{0}=10^{4}$ then $\vartheta^{T} \simeq 10^{-6} \div 10^{-7} L^{3}$ and, consequently, for $\mathrm{L}=1 \mathrm{~cm}$ we will have the rotation angle $\vartheta^{T} \simeq 10^{-6} \div 10^{-7} \mathrm{rad}$.

As it is seen, we have obtained the T-violating rotation angle $\vartheta^{T}$ of the same order of $\vartheta^{P}$. It makes possible experimental observation of the phenomenon of the T-violating polarization plane rotation.

It should be noted that the manufacturing of diffraction gratings for the range being more longwave than the visible light one may be simpler. That is why we would like to attract attention to the possibility of studying of the T-violating polarization plane rotation in the vicinity of frequencies of atomic (molecular) hiperfine transitions; for example, for $\mathrm{Ce}$ (the transition wavelength is $\lambda=3.26 \mathrm{~cm})$ and $\mathrm{Tl}(\lambda=1.42 \mathrm{~cm})$.

\section{Conclusion}

Thus, we have shown that the phenomenon of the T-violating polarization plane rotation appears while the photon is scattered by a volume diffraction grating. The phenomenon grows sharply in the vicinity of the resonance transmission condition. An experimental scheme based on a waveguide, containing a diffraction grating and gas, has been proposed which enables real experiments on observation of the T-violating polarization plane rotation to be performed. The rotation angle has been shown to be $\vartheta^{T}=10^{-6} \div 10^{-7} L^{3}$, where $\mathrm{L}$ is the waveguide length (thickness of the equivalent volume diffracting grating). Let there is the electric field in the region of the gas. As a result, the scattering amplitude contains the T-violating term $f_{E}^{T}(0)=i \beta_{E}^{T} \vec{E}\left[\vec{e}^{*} \times \vec{e}\right]$. The phenomenon of the T-violating photon polarization plane rotation about $\vec{E}$ appeares due to this term. The effect grows sharply in the volume diffraction grating too, but the diffraction grating can be centrisymmetrical. More over the effect can be observed in usual resonator or ring laser because the sign of the photon polarization plane rotation about $\vec{E}$ do not depend from the direction of the photon momentum $\vec{k}$ unlike the P-violating, T-even polarization 
plane rotation effect which is proportional to $i \beta_{v}^{P} \vec{n}\left[\vec{e}^{*} \times \vec{e}\right]$.

\section{Neutron spin rotation and spin dichroism in media.}

Neutron spin rotation and spin dichroism in media caused by parity $(\mathrm{P})$ violation and possible time $(\mathrm{T})$ noninvariance under neutron interaction with nuclei are being actively explored recently (Alfimenkov (1983), Sushkov,Flambaum (1982), Stodolsky (1982), Koster et al (1991), Baryshevsky (1983), Bowman et al (1990), Franke et al (1991)). The mentioned effects are described by the refractive index of neutrons in media

$$
\hat{N}=1+\frac{2 \pi \rho}{k^{2}} \hat{f}(0),
$$

where $k$ is the neutron wave number, $\rho$ is the scatters density, $\hat{f}(0)$ is the coherent elastic forward scattering amplitude of neutrons on a nucleus.

M.Forte shows (Forte (1983), Forte, Zayen (1989)) that the T-noninvariant effect of spin rotation arises under neutron diffraction by crystals with nonpolarized nuclei. This effect is determined by coherent elastic scattering amplitude at a non-zero angle $\hat{f}\left(\vec{k}^{\prime}, \vec{k}\right) \sim \vec{\sigma}\left(\vec{k}^{\prime}-\vec{k}\right)$, where $\vec{\sigma}$ is the Pauli matrix describing neutron spin, $\vec{k}^{\prime}$ is the wave vector of scattered neutron.

In (Forte (1983), Forte, Zayen (1989), Fedorov, Voronin, Lapin (1992)) the possibility of experimental measurements of this spin rotation effect was considered. One of the main difficulties of the diffraction methods suggested in (Forte (1983), Forte, Zayen (1989), Fedorov, Voronin, Lapin (1992)) is that even for thermal neutrons the beams with very low

angular divergence rad and very high degree of monochromaticity $\frac{\Delta k}{k} \sim \frac{\Delta v}{v_{B}} \sim 10^{-5} \div 10^{-6}$ are needed, where $v_{B}$ is the angle of Bragg diffraction. Only very small part $\Delta \Phi$ of a neutron flow $\Phi$ can be used to carry out the experiment: $\Delta \Phi \sim \Phi \frac{\Delta k}{k} \Delta v$. The situation is still worse for the range of epithermal neutrons in which $\Delta v \sim 10^{-7} \mathrm{rad}$ and even lower.

In (Baryshevsky (1995)) we have shown that there are new effects: the effects of Tnoninvariant spin rotation and spin dichroism in the nonpolarized crystals even in case when diffraction conditions are not fulfilled. As a consequence, not only flow $\Delta \Phi$ increases, but the possibility of investigation of in $\hat{f}\left(\vec{k}^{\prime}, \vec{k}\right)$ the range of epithermal neutrons arises.

So, let the neutrons move through a crystal. According to (Baryshevsky (1995), Baryshevsky (1976), Baryshevsky, Cherepitsa (1985)) the Schrodinger equation describing propagation of a coherent neutron wave $\Psi$ in a crystal with polarized nuclei in the impulse representation has the view

$$
\left(k^{2}-k_{0}^{2}\right) \Psi(\vec{k})+\sum_{\vec{\tau}} \frac{2 m}{\hbar^{2}} \hat{U}_{e f f}(\vec{\tau}) \Psi(\vec{k}-2 \pi \vec{\tau})=0,
$$

where $k_{0}$ is the neutron wave number in vacuum, $k$ is the neutron wave number in a crystal. The Fourie transform of neutron effective potential energy in a crystal is

$$
\hat{U}_{e f f}(\vec{\tau})=-\frac{2 \pi \hbar^{2}}{m V_{0}} \hat{F}(\vec{\tau}) ; \hat{F}(\vec{\tau})=\sum_{j} \hat{f}_{j}(\vec{\tau}) e^{-w_{j}(\vec{\tau})} e^{-i 2 \pi \vec{\tau} \vec{R}_{j}}
$$


$\hat{F}(\vec{\tau})$ is the amplitude of neutron coherent scattering on crystal unit cell in the direction $\vec{k}^{\prime}=\vec{k}+2 \pi \vec{\tau}$, where $2 \pi \vec{\tau}$ is the vector of crystal reciprocal lattice, $\hat{f}_{j}(\vec{\tau})$ is the amplitude of coherent elastic scattering $\hat{f}_{j}\left(\vec{k}^{\prime}, \vec{k}\right)$ on the nuclei of j-type in the direction $\vec{k}^{\prime}=\vec{k}+2 \pi \vec{\tau}$ , $\vec{R}_{j}$ is the coordinate of the $\mathrm{j}$ nucleus in the unit cell, $e^{-w_{j}(\vec{\tau})}$ is the Debay-Waller factor.

The system of homogeneous equations (2.2) permits to determine the dependence of $k$ on $k_{0}$, i.e. to determine the neutron refractive index in a crystal.

It is well-known that the area $\Delta v$ of neutron incidence angle on a crystal in which strong diffraction is observed (when the diffracted wave amplitude is comparable with the amplitude of incident wave) is very small $\Delta v \sim 10^{-5} \div 10^{-6}$ rad even for thermal neutrons.

Outside this narrow angle area the diffracted wave amplitude is small and the system of equations (2.2) can be analyzed according to the perturbation theory. As a result, we have

$$
\hat{N}=\frac{k}{k_{0}} \simeq 1+\frac{1}{2} \hat{g}(0)+\frac{\hat{g}(-\vec{\tau}) \hat{g}(\vec{\tau})}{2 \alpha_{B}}
$$

where

$$
\hat{g}(\vec{\tau})=-\frac{2 m}{\hbar^{2} k_{0}^{2}} \hat{U}_{e f f}(\vec{\tau}), \alpha_{B}=\frac{2 \pi \vec{\tau}\left(2 \pi \vec{\tau}+2 \vec{k}_{0}\right)}{k_{0}^{2}}
$$

As we see, the correction to the neutron refractive index in crystals contains the scattering amplitude at the non-zero angle $\hat{f}(\vec{\tau})$.

According to (Baryshevsky (1995)) the expression for $\hat{g}(\vec{\tau})$ can be written as a sum

$$
\hat{g}(\vec{\tau})=\hat{g}_{s}(\vec{\tau})+\hat{g}_{s o}(\vec{\tau})+\hat{g}_{w}(\vec{\tau}),
$$

where $\hat{g}_{s}(\vec{\tau})$ is proportional to the scattering amplitude on the nucleus $\hat{f}_{s}(\vec{\tau})$ due to strong interactions minus spin-orbital scattering interactions; $\hat{g}_{w}(\vec{\tau})$ is proportional to the $\mathrm{P}$ and T-violating amplitude; $\hat{g}_{s o}=\hat{g}_{s o n}+\hat{g}_{s h w}, \hat{g}_{s o n}$ is proportional to the amplitude of spinorbital neutron scattering on the nucleus due to nuclear forces $\hat{f}_{\text {son }}(\vec{\tau})=f_{\text {son }} \vec{\sigma}[\vec{k} \times 2 \pi \vec{\tau}]$, $\hat{g}_{s h w}$ is proportional to the amplitude of spin orbital neutron scattering on the nucleus due to neutron magnetic momentum interaction with the nucleus electric field (the so-called Schwinger scattering):

$$
\hat{g}_{s h w}=i \frac{2 m}{V_{0} \hbar^{2} k_{0}^{2}} \frac{\mu \hbar}{m c} \sum_{j} \Phi_{j}(\vec{\tau}) e^{-w_{j}(\vec{\tau})} \vec{\sigma}[\vec{k} \times 2 \pi \vec{\tau}] e^{-i 2 \pi \vec{\tau} \vec{\tau}_{j}},
$$

$\mu$ is the magnetic neutron momentum, $\Phi_{j}(\vec{\tau})$ is the Fourie component of electrostatic potential induced by nucleus $j$.

In case of the target with nonpolarized nuclei $\hat{g}_{s}(\vec{\tau})=g_{s}(\vec{\tau})$ does not depend on neutron spin. Besides, for slow neutrons $g_{s o} \ll g_{s}$. It allows to write the contribution to caused by diffraction in the following way

$$
\begin{aligned}
\delta \hat{N}=\frac{\hat{g}(-\vec{\tau}) \hat{g}(\vec{\tau})}{2 \alpha_{B}} & =\frac{g_{s}(-\vec{\tau}) g_{s}(\vec{\tau})}{2 \alpha_{B}}+\frac{1}{2 \alpha_{B}} g_{s}(-\vec{\tau})\left[\hat{g}_{s o}(\vec{\tau})+\hat{g}_{w}(\vec{\tau})\right]+ \\
& +\frac{1}{2 \alpha_{B}} g_{s}(\vec{\tau})\left[\hat{g}_{s o}(-\vec{\tau})+\hat{g}_{w}(-\vec{\tau})\right] .
\end{aligned}
$$


Let the target be nonpolarized. In this case T-noninvariant part of $\hat{g}(\vec{\tau})$ is (Baryshevsky (1995))

$$
\begin{gathered}
\hat{g}_{w}^{T}(\vec{\tau})=\frac{4 \pi}{k^{2} V_{0}} \sum_{j} \hat{f}_{j w}^{T}(\vec{\tau}) e^{-w_{j}(\vec{\tau})} e^{-i 2 \pi \vec{\tau} \vec{R}_{j}}, \\
\hat{f}_{j w}^{T}(\vec{\tau})=C_{j}^{\prime}(\vec{\tau}) \vec{\sigma} \frac{\vec{\tau}}{\tau}, C_{j}^{\prime}(\vec{\tau})=\operatorname{Re} C_{j}^{\prime}+\operatorname{Im} C_{j}^{\prime} .
\end{gathered}
$$

From $(2.7,2.8)$ we see that the T-noninvariant contribution to the refractive index in a nonpolarized crystals may occur only in a noncentrosymmetric crystals.

We see also that we have the two effects: the difference of the absorption coefficient of the neutrons with spin parallel and antiparallel to the vector $\vec{\tau}$ (spin dichroism) and the spin rotation around of the direction of $\vec{\tau}$.

Difference $æ_{\uparrow \uparrow}-æ_{\downarrow \uparrow}=k\left(\operatorname{Im} \delta N_{\uparrow \uparrow}-\operatorname{Im} \delta N_{\downarrow \uparrow}\right)$

Spin rotation angle $v=k R e\left(\delta N_{\uparrow \uparrow}-\delta N_{\downarrow \uparrow}\right) L$

Difference of the neutrons number $J_{\uparrow \uparrow}$ with spin parallel to $\vec{\tau}$ and the neutrons number $J_{\downarrow \uparrow}$ with spin antiparallel to $\vec{\tau}$ which passed through the crystal is

$$
\frac{J_{\uparrow \uparrow}-J_{\downarrow \uparrow}}{J_{\uparrow \uparrow}-J_{\downarrow \uparrow}}=k \operatorname{Im}\left(\delta N_{\uparrow \uparrow}-\delta N_{\downarrow \uparrow}\right) L
$$

where $L$ is the length of the neutron way in the crystal.

From (2.7) it follows that at the constant $\alpha_{B}$ the angle $v$ decreases proportionally to $k^{-3}$ and absorption $\sim k^{-1}$ with the neutron energy increasing. So, at first glance the transfer to the epithermal range only makes worse the situation. However, it should be taken into account that in the range of resonances the amplitudes $f_{w}(\vec{\tau})$ sharply increase due to amplification mechanisms, being analogous to the well-known ones for the scattering amplitudes (Forte (1983), Baryshevsky (1995)). According to (Alfimenkov (1983)) there are two amplification mechanisms: dynamical and kinematic. They lead to the increase of the scattering amplitude near resonance in $\sqrt{\frac{\Delta E}{D}} \cdot \sqrt{\frac{\Gamma_{n s}}{\Gamma_{n p}}} \simeq 10^{5} \div 10^{6}$, where $\Delta E$ is the averaged distance between the single particle nuclei states, $D$ is the averaged distance between the levels of the compound nucleus, $\Gamma_{n s(p)}$ are the neutron widths of $s(p)$ the neutron state in the incident wave. As a result, for the neutrons with the energies $1-10 \mathrm{ev}$ we can expect the effect increase in $10^{3} \div 10^{2}$ times and as a consequence the decrease in limitation for the detection of $\mathrm{T}$ - noninvariant effect. It is very important that neutron part $\Delta \Phi$ even increases as the magnitude $\frac{\Delta k}{k} \sim \frac{\Delta v_{B}}{v_{B}}$ and for $\Delta v_{B}=10^{-4}$, $v_{B}=\frac{2 \pi \tau}{k} \sim 10^{-1}$ we have ten time increase of $\Delta \Phi$. At the same time investigation methods of spin rotation in the field of strong diffraction for the neutrons with such an energy are practically unrealized, since angular range of strong diffraction becomes $\Delta v_{B} \sim 10^{-7}$ rad. Two reasons simultaneously spoil the effect: angular spread of crystal mosaic structure $\left(\Delta \epsilon_{m} \geq 10^{-6} \mathrm{rad}\right)$ and sharp suppression of flux $\Delta \Phi$ in such a small angular and energetic interval $\Delta v_{B} \frac{\Delta k}{k} \sim \frac{\Delta v_{B}^{2}}{v_{B}}=10^{-13}$.

It is very important to say that the difference of absorption coefficient changes with the neutron energy increasing more slowly $\left(\sim k^{-1}\right)$ than $v$ and sharply increases in the range of resonances also. As a result the study of the effect of spin dichroism may give the essential advantage in epithermal energy range. 


\section{References}

To part 1.

Christenson J.H., Cronin J.W., Fitch V.L. and Turlay R. Phys. Rev. Lett. 13 (1964) 1138

V.G.Baryshevsky Phys. Lett A 177 (1993) 38

V.G. Baryshevsky and D.V.Baryshevsky Journ of Phys. B: At. Mol. Opt. Phys. 27 (1994) 4421

A.M.Barkov and N.S.Zolotariov Lett. J. Exp. Theor. Phys. 118 (1978) 379 (in Russian)

M.A.Bouchiat and L.Pottier Science 234 (1986) 1203

V.V.Fedorov, V.V.Voronin, and E.G.Lapin Journ. of Phys. G: Nucl.Part. Phys. G 18 (1992) 1133

M.J.Forte Journ. of Phys. G.: Nucl. Part. Phys. G 9 (1983) 745

J.D.Jackson Classical electrodinamics (John Wiley \& Sons, INC, New York - London) (1962)

I.V.Khriplovich Party Nonconservation in Atomic Phenomena 1991 (London: Gordon and Breach)

S.K.Lamoreaux Nucl. Instrum. Methods A 284 (1989) 43

S.A.Maksimenko, G.Ya.Slepyan Electromagnetics 17 (1997) 147

Shih-Lin Chang Multiple Diffraction of X-rays in Crystals 1984 (Springer-Verlag Berlin Heidelberg New York Tokyo)

T.Tamir Guided-Wave Optoelectronics 1988 (Springer Verlag New York)

To part 2 .

V.P.Alfimenkov, Nuclear Physics A398 (1983) 93.

O.Sushkov, V.Flambaum, Usp.Fiz.Nauk 25 (1982) 1.

L.Stodolsky, Phys.Lett. B197 1982) 213.

J.E.Koster et al, Phys.Lett B267 (1991) 23.

V.G.Baryshevsky, Sov.J.Nucl.Phys. 38 (1983) 699.

J.Bowman et al, Phys.Rev.Lett.65 (1990) 1192.

C.Franke et al, Phys. Rev.Lett 67 (1991) 564.

M.Forte, J. of Phys.G 9 (1983) 745.

M.Forte, C.Zayen, Nucl. Instr. Met. A284 (1989) 147.

V.Fedorov, V.Voronin, E.Lapin, J. of Phys. Journ. of Phys. G: Nucl.Part. Phys. G 18, (1992), 1133

V.G.Baryshevsky, Physics of Atomic Nuclei 58 (1995) 1471

V.G.Baryshevsky, J Exp.Theor Phys. 70 (1976) 430

V.G.Baryshevsky, S.V.Cherepitsa, Phys.Stat.Sol (b) 128 (1985) 379

L.I.Lapidus, Rev.Nod.Phys. 39 (1967) 689 